PAPER

\title{
MRI directed bilateral stimulation of the subthalamic nucleus in patients with Parkinson's disease
}

\author{
N K Patel, P Plaha, K O'Sullivan, R McCarter, P Heywood, S S Gill
}

J Neurol Neurosurg Psychiatry 2003;74:1631-1637

See end of article for authors' affiliations .....................

Correspondence to: Dr S S Gill, Consultant Neuroarsurgeon, Department of

Neurosurgery, Frenchay Hospital, Bristol BS16 1LE, UK; steven.gill@north-bristol. swest.nhs.uk

\begin{abstract}
Objective: Bilateral chronic high frequency deep brain stimulation (DBS) of the subthalamic nucleus (STN) has emerged as an appropriate therapy for patients with advanced Parkinson's disease refractory to medical therapy. Advances in neuroimaging and neurophysiology have led to the development of varied targeting methods for the delivery of this treatment. Intraoperative neurophysiological and clinical monitoring is regarded by many to be mandatory for accurate STN localisation. We have examined efficacy of bilateral STN stimulation using a predominantly magnetic resonance imaging (MRI)-directed technique.

Methods: DBS leads were stereotactically implanted into the STN using an MRI directed method, with intraoperative macrostimulation used purely for adjustment. The effects of DBS were evaluated in 16 patients followed up to 12 months, and compared with baseline assessments. Assessments were performed in both off and on medication states, and were based on the Unified Parkinson's Disease Rating Scale (UPDRS) and timed motor tests. Functional status outcomes were examined using the PDQ-39 quality of life questionnaire. A battery of psychometric tests was used to assess cognition.

Results: After 12 months, stimulation in the off medication state resulted in significant improvements in Activities of Daily Living and Motor scores (UPDRS parts II and III) by $62 \%$ and $61 \%$ respectively. Timed motor tests were significantly improved in the off medication state. Motor scores (UPDRS part III) were significantly improved by $40 \%$ in the on medication state. Dyskinesias and off duration were significantly reduced and the mean dose of L-dopa equivalents was reduced by half. Psychometric test scores were mostly unchanged or improved. Adverse events were few.

Conclusions: An MRI directed targeting method for implantation of DBS leads into the STN can be used safely and effectively, and results are comparable with studies using intraoperative microelectrode neurophysiological targeting. In addition, our method was associated with an efficient use of operating time, and without the necessary costs of microelectrode recording.
\end{abstract}

$\mathrm{D}$ uring the past decade, there has been resurgence in the neurosurgical treatment of selected patients with advanced Parkinson's disease (PD) who become poorly controlled despite optimised medical therapy, and in particular suffer from motor fluctuations and drug-induced dyskinesias.

Deep brain stimulation (DBS) has become an accepted technique for the treatment of several movement disorders and in particular for PD. ${ }^{1-3}$ It was first applied to the ventral intermediate nucleus of the thalamus ${ }^{45}$ to treat tremor, but more recently, the surgical target of interest has moved to the subthalamic nucleus (STN) and the internal globus pallidus, because stimulation in these structures is additionally able to improve bradykinesia and akinesia in PD. ${ }^{6-8}$ Although there are few comparative data between these two targets, most groups consider that STN is the optimal target for patients with PD requiring surgery.

The most appropriate targeting method to place DBS leads accurately and effectively in STN remains a subject of controversy. Typically, a combination of anatomical and physiological methods is used in the localisation of the STN. Anatomical methods include both direct and indirect techniques. ${ }^{9}$ Using imaging acquired in stereotactic conditions, the coordinates of the STN may be obtained directly from the image if the nucleus can be adequately visualised; alternatively, an indirect method is used, employing atlasbased coordinates generated in proportion to recognised internal landmarks, such as the anterior-posterior commissural distance or third ventricular dimensions. Physiological methods include intraoperative macrostimulation and micro-electrode recording. Many groups continue to use an indirect method with atlas-based coordinates, but "fine tune" localisation with intraoperative clinical and electrophysiological monitoring procedures. ${ }^{6}{ }^{10}$ The main points of dispute concern the need, benefit, and morbidity of intraoperative microelectrode recording for target localisation and the need for direct $v$ indirect anatomical methods of target localisation. In this paper, we report an interim 12 month analysis of 16 patients with idiopathic PD who underwent bilateral DBS of the STN using a direct anatomical magnetic resonance imaging (MRI) directed method with intraoperative macrostimulation used for confirmation of target localisation, but without intraoperative microelectrode recording.

\section{CLINICAL MATERIALS AND METHODS \\ Patient population}

The study population comprises the first 16 consecutive patients (10 men, six women) to have undergone bilateral STN DBS at our centre and who have now completed a 12 month follow up evaluation. The mean age (SD) of the patients at the time of surgery was 56 (11) years, and mean (SD) disease duration was 10 (3.5) years. The selection criteria were that the patient should have L-dopa responsive idiopathic PD with severely disabling symptoms despite best medical therapy. In addition, the patients had to be able to

Abbreviations: DBS, deep brain stimulation; MRI, magnetic resonance imaging; PD, Parkinson's disease; PDQ-39, 39-item Parkinson's disease quality of life questionnaire; STN, subthalamic nucleus 
function at a reasonable level of independence for at least some part of the average day. Patients with a significant history of depression, evident cognitive impairment based on neuropsychological testing (operationally defined as scores on tests of two or more cognitive domains (McCarter et al) being greater than 1.5 standard deviations below the population mean) and those deemed medically unfit were excluded from the study. Typically our patients had periods of severe immobility for about $50 \%$ of the day, and suffered from on dyskinesia for $30-40 \%$ of the remaining day (fig 6).

\section{Surgery}

All patients gave informed consent. The STN was localised with high-resolution MRI T2 scan sequences (1.5 Tesla TR 2500, TE 150, TSE 11, NSA 12) and per-operative macrostimulation was used to refine accuracy of targeting. Under general anaesthesia, a modified Leksell stereotactic frame was affixed parallel to the orbitomeatal plane. The anterior (AC) and posterior (PC) commissures were identified in a mid-sagittal planning scan. Axial images (fig 1), 2 mm thick, were acquired parallel to the AC-PC plane, and coronal images orthogonal to these were then obtained. We have found that these sequences give optimum delineation of the STN and related structures. We used magnified hard copies of the MRI scans and overlaid the T2 scans with inverted T2 images (fig l) to enhance the definition of STN boundaries further. The Schaltenbrand atlas ${ }^{11}$ was used as a visual guide in defining the boundaries of STN and its surrounding structures. We selected the dorsolateral STN as our target, which is functionally implicated in sensorimotor circuits. ${ }^{12}$ The target was centred in the posterior third of the STN. The coordinates of the target were defined, and a trajectory was planned in the coronal plane, avoiding superficial blood vessels, and running through the caudate nucleus and lateral thalamus, aiming for the centre of the dorsal half of the posterior STN (fig 2).

The Leksell frame with its in-house modifications is relocatable, and in the initial number of cases in this cohort, the frame was usually removed after the MRI scan and relocated the following day under general anaesthesia, using CT to confirm accuracy of relocation, prior to waking the patient up for the first part of the surgical procedure. It was subsequently found that patients could tolerate the frame overnight, avoiding the need for relocation and its anaesthetic, and this has now become our routine practice.

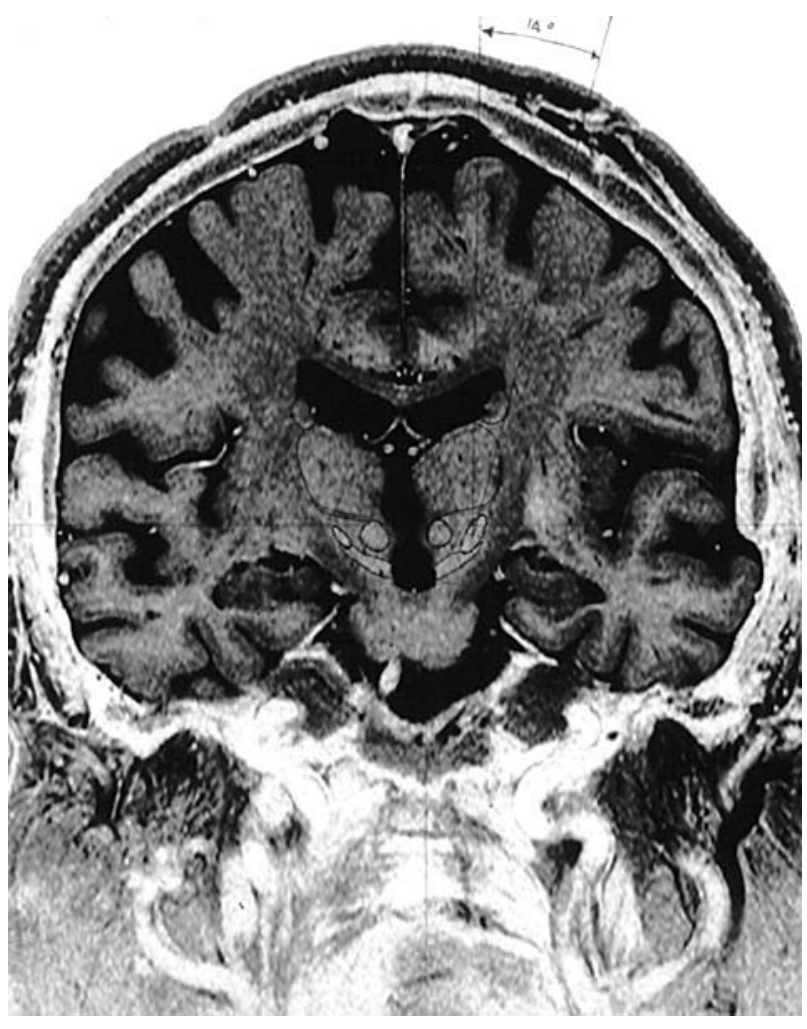

Figure 2 High resolution coronal inverted T2 weighted MR images with trajectory planned down STN axis and avoiding superficial blood vessels.

At surgery, the following day, simultaneous bilateral DBS leads were implanted with patients awake and in an 'off' state, antiparkinsonian medications having been stopped $12 \mathrm{~h}$ previously. Surgery was performed with the patients in a sitting position, and with constant saline irrigation of the burrhole to avoid air entering the cranium and therefore minimizing brain shift. A $1.24 \mathrm{~mm}$ diameter electrode with a $2 \mathrm{~mm}$ exposed tip (Radionics Inc., Burlington, MA, USA) was guided to the dorsolateral STN. The target was stimulated at $100 \mathrm{~Hz}, 0.75-2 \mathrm{~V}$, with $1 \mathrm{~ms}$ pulse width, during which changes in tremor, rigidity, and bradykinesia were monitored.
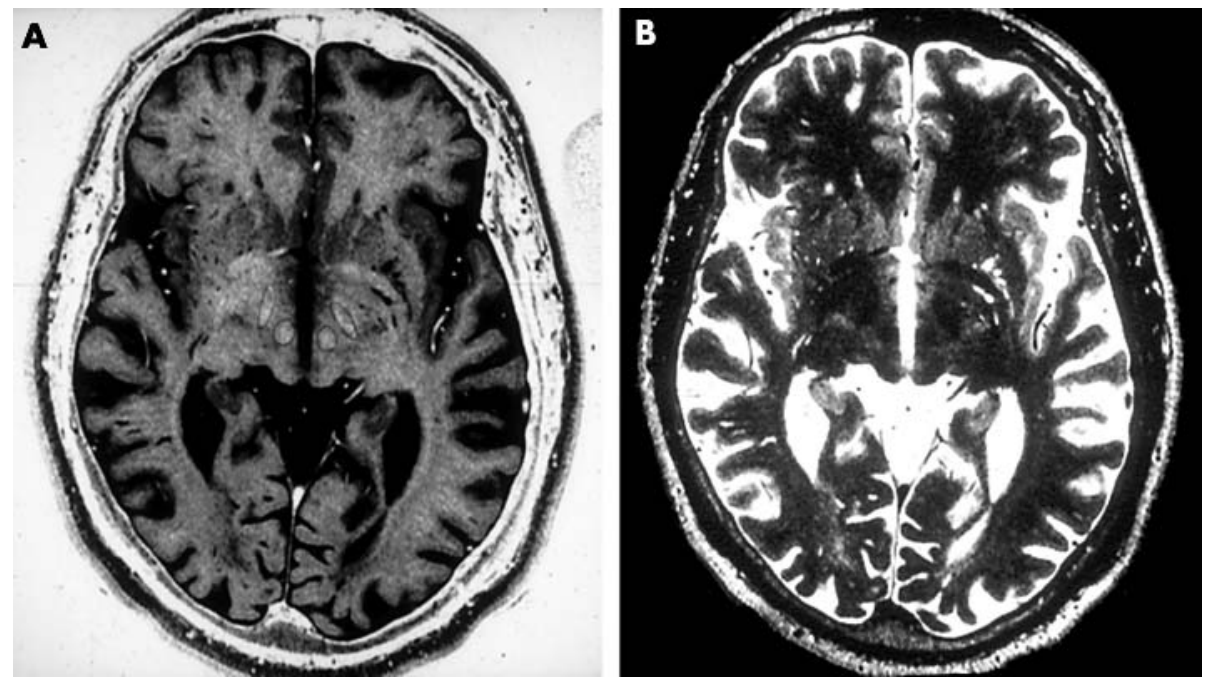

Figure 1 High resolution axial T2 weighted and inverted T2 weighted MR images with bilateral STN and red nuclei delineated on inverted image, with evident individual variability. 
The depth of the probe was adjusted to gain maximum clinical improvement without the development of side effects. The optimal position for stimulation was recorded, and the stimulating electrode removed and replaced with a DBS lead (model DBS 3389; Medtronic Inc., MN, USA), which was advanced so that at least one contact was positioned below the desired target. The lead was secured to the skull with a mini-plate and screws and the $1 / 4$ inch burrhole sealed with acrylic cement.

When both DBS leads had been implanted, the patient was anaesthetised and the DBS pulse generator (Kinetra; Medtronic Inc.) implanted in a subcutaneous pocket below the clavicle. Extension leads were tunnelled between the infraclavicular and scalp openings, and connected the DBS leads to the pulse generator.

Implantation of the DBS leads typically took on average between 30-45 min per side, and implantation of the Kinetra generator and connection leads a further 60-90 mins (total time for all the implants about $3 \mathrm{~h}$ ).

\section{Electrical stimulation setting and medical therapy}

The Kinetra generator was switched on immediately or within days of surgery, with antiparkinsonian medications reduced by $50 \%$ and then re-adjusted as required. In the days following surgery, the effects of stimulation were assessed for each of the four lead contacts, and the optimal settings were programmed. Initially, a monopolar electrode setting was used, and subsequently a bipolar setting if a more focal stimulation, maintaining effect without side effects, was required. Patients were discharged generally between days 4-7 postoperatively, with preset stimulation parameters at the optimal lead contacts, and were advised to increase the stimulation amplitude and reduce medication as required, titrating to optimise effect and avoid side effects. Patients were able to contact a specialist PD nurse for advice over the phone, and were reviewed in the clinic at 6 weeks and 6 monthly thereafter.

\section{Clinical evaluation}

Evaluations were performed preoperatively and at 12 months postoperatively. Clinical evaluations were based on the Core Assessment Program for Intracerebral Transplantations, a validated protocol for evaluating surgical treatments of idiopathic $\mathrm{PD},{ }^{13}$ and included the Unified Parkinson's Disease Rating Scale (UPDRS), the Hoehn and Yahr scale, and timed motor tests. Patients were assessed in two conditions before surgery (off and on medication) and in four conditions after surgery (off medication, off stimulation; off medication, on stimulation; on medication, off stimulation; and on medication, on stimulation). Patients were assessed in the off state, having stopped their medication at least $12 \mathrm{~h}$ beforehand, and had their stimulation switched off overnight. The same assessments were then repeated on medication after administration of $200 \mathrm{mg}$ L-dopa (plus $50 \mathrm{mg}$ of peripheral dopa-decarboxylase inhibitor), with all patients having had a protein-free breakfast on the morning of the assessment.

In addition, pre- and post-operative neuropsychological assessments of attention, memory, executive function, language, and verbal intellect were undertaken with a battery of tests designed to minimise potential contamination of cognitive effects by motor symptoms. ${ }^{14}$ One patient did not complete both pre- and post-operative testing, and is excluded from the cognitive analysis.

\section{Statistical analysis}

The primary outcome measures were the scores on parts II (Activities of Daily Living) and III (Motor examination) of the UPDRS. The secondary measures were as follows: the subscores on part III of the UPDRS; subscores on part IV (complications of therapy) of the UPDRS; the Hoehn and Yahr global stage; timed motor tests, L-dopa equivalent requirements; patient diaries; and quality of life as measured by the PDQ-39.

The data were analyzed using the paired Wilcoxon signedrank and paired Student's $t$ test.

The psychometric data were analysed using the Reliable Change Index. ${ }^{15}$ This robust test is increasingly used as it often provides a more clinically meaningful method of analysing change in neuropsychological performance than traditional measures of statistical significance or group differences. The latter may mask significant individual change in groups of patients who may show heterogeneous outcomes, as described after surgery for PD. ${ }^{16}{ }^{17}$

\section{RESULTS}

All 16 patients, who have been followed for at least 12 months, have received sustained benefit from the procedure. The effect of stimulation on the patients' motor performance (UPDRS part III) was significant in both the off and on medication states, resulting in a $61 \%(\mathrm{p}<0.001)$ and $40 \%(p=0.007)$ reduction respectively (fig 3$)$. Long-term stimulation resulted in improved scores for akinesia, rigidity, tremor, impairment of arising from chair, gait and postural instability, when patients were evaluated off medication. In the on medication state, stimulation significantly improved tremor scores (table 1).

The effect of stimulation on bradykinesia was also assessed based on changes shown in standardised timed motor tests. All the timed motor tests showed significant improvements with stimulation in the off medication state (table 2). Five patients were unable to complete the stand/walk test while in the off medication state preoperatively, but were able to accomplish the task postoperatively with stimulation on.

There was significant improvement in the patients' functional performance with stimulation on, as demonstrated by improvements in the activities of daily living scores (UPDRS part II), which were improved by $62 \%(\mathrm{p}=0.001)$ (fig 4). With stimulation on, Hoehn and Yahr scores (for global stage of disease) were significantly improved in both off $(p<0.001)$ and on $(p=0.006)$ medication states (table 1$)$.

Stimulation significantly reduced dyskinesias and motor fluctuations based on complications of therapy scores (UPDRS part IV) (fig 5). The mean (SD) score for the duration of the off period was reduced from 2.2 (1.3) before surgery to $1.0(1.2)$ at 12 months $(\mathrm{p}=0.007)$ (UPDRS part IV,

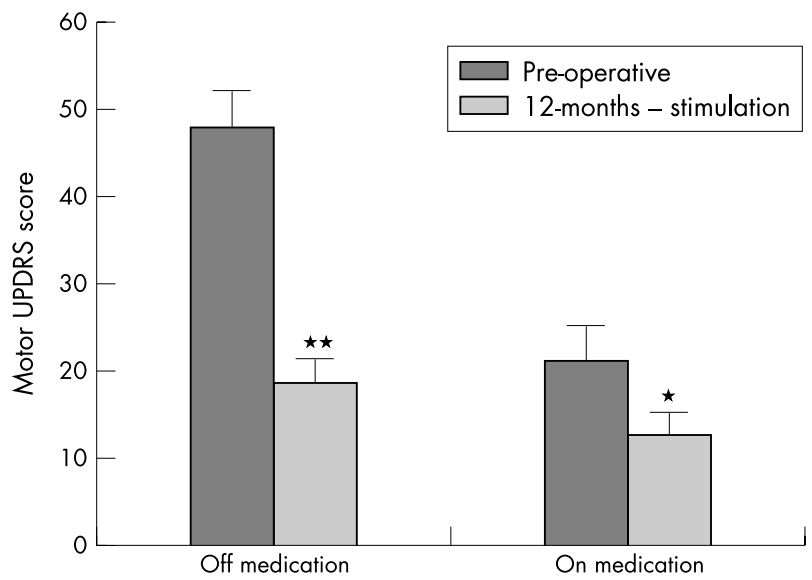

Figure 3 Mean (SD) off and on-medication scores for motor performance (UPDRS part III) at baseline and at 12 months after surgery with stimulation on. ${ }^{*} p=0.007 ;{ }^{* *} p<0.001$, for comparison with the same condition before surgery. 
Table 1 Unified Parkinson's Disease Rating Scale motor scores and L-dopa equivalent medication requirement in patients following bilateral deep brain stimulation of the subthalamic nucleus

\begin{tabular}{|c|c|c|c|c|}
\hline & \multicolumn{2}{|c|}{ Pre-operation } & \multicolumn{2}{|c|}{12 months post-operation } \\
\hline & Med off & Med on & Med off & Med on \\
\hline UPDRS III & $47.8(4.0)$ & $21.1(3.9)$ & $18.6(2.7)^{\star * *}$ & $12.8(2.3)^{* * *}$ \\
\hline Rigidity & $10.9(1.4)$ & $3.9(1.1)$ & $3.1(0.7)$ & $1.9(0.6)$ \\
\hline Bradykinesia & $18.9(1.8)$ & $8.0(1.6)$ & $9.8(1.2)$ & $6.9(1.2)$ \\
\hline Tremor & $6.7(1.3)$ & $2.6(1.0)$ & $0.6(0.4)^{* * *}$ & $0.3(0.2)^{*}$ \\
\hline Arising from chair & $2.1(0.4)$ & $0.4(0.2)$ & $0.6(0.2)^{* *}$ & $0.3(0.2)$ \\
\hline Gait disturbance & $2.1(0.3)$ & $0.6(0.2)$ & $0.7(0.2)^{\star *}$ & $0.4(0.2)$ \\
\hline Postural instability & $2.1(0.4)$ & $0.8(0.2)$ & $0.8(0.3)^{\star \star}$ & $0.5(0.2)$ \\
\hline Speech & $1.7(0.2)$ & $1.3(0.2)$ & $1.3(0.2)^{* *}$ & $1.2(0.2)$ \\
\hline $\begin{array}{l}\text { Global stage of disease } \\
\text { (Hoehn and Yahr) }\end{array}$ & $4.1(0.3)$ & $2.7(0.3)$ & $2.5(0.2)^{\star \star *}$ & $2.1(0.2)^{* *}$ \\
\hline Levodopa (mg) & \multicolumn{2}{|c|}{$856.4(158.2)$} & \multicolumn{2}{|c|}{$443.9(82.5)^{\star *}$} \\
\hline
\end{tabular}

Values are means (SEM).

For all scores, a reduction indicates an improvement in function.

UPDRS III denotes motor scores, items $18-31$, maximal points $=108$; tremor subscores, items 20 and 21, maximal points $=28$; rigidity subscores, item 22 , maximal points $=20$; bradykinesia subscores, items $23-26$, maximal points 32

${ }^{*} \mathrm{p}<0.05,{ }^{* *} \mathrm{p}<0.01$; ${ }^{* * *} \mathrm{p}<0.001$, compared with the condition before surgery.

item 39; range 0-4). The mean score for the duration of dyskinesias decreased from $1.3(1.1)$ to $0.7(0.5)(\mathrm{p}<0.02)$ (UPDRS part IV, item 32; range $0-4$ ). The mean disability related to dyskinesias decreased from 1.6 (1.4) to $0.2(0.5)$ $(p<0.01)$ (UPDRS part IV, item 33; range 0-4). The duration of the on period increased correspondingly (fig 6). An attempt to use patient diaries to record the durations of on and off periods was unsuccessful because of poor compliance and data entry.

\section{Medications and electrical treatment}

At 12 months, the mean daily dose of L-dopa equivalents based on a formula as designated by Pahwa et al ${ }^{18}$ decreased significantly by $48 \%(p=0.002)$ (table 1$)$. All patients were being stimulated continuously throughout the whole day. Seven patients were receiving bilateral monopolar stimulation, six were receiving bipolar stimulation, and three were receiving bipolar stimulation on one side and monopolar stimulation on the other. The frequency was between 130 and $180 \mathrm{~Hz}$ and the pulse width was between 60 and $90 \mu \mathrm{s}$. The middle two contacts on the quadripolar electrode were shown to be most effective in $90 \%$ of cases.

\section{Functional status (PDQ-39)}

Completed questionnaires were received for 14 out of the 16 patients (results shown in table 3). The Activities of Daily Living and Stigma dimensions were significantly lower at 12 months $(\mathrm{p}<0.02$ and $\mathrm{p}<0.03$ respectively), as was the PDQ-39 summary index $(\mathrm{p}<0.01)$. The six other domains of the PDQ-39 were not significantly modified by the procedure; however, non-significant reductions were evident for the

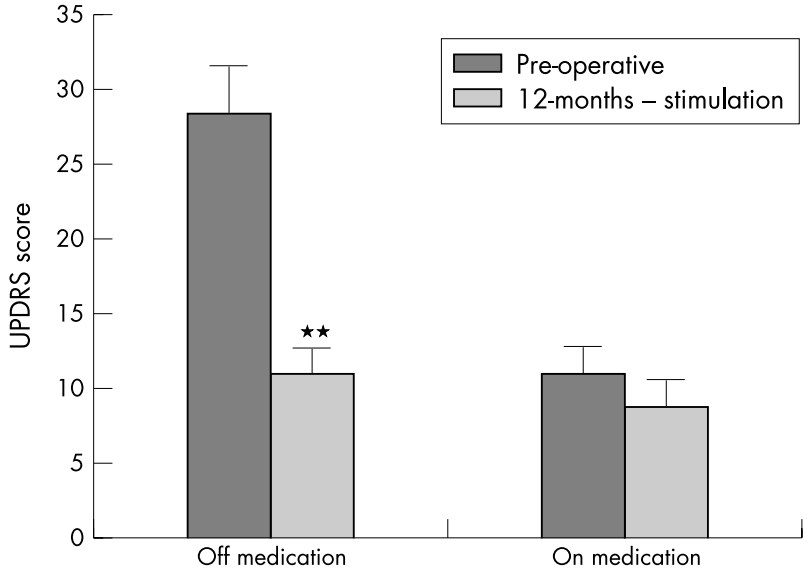

Figure 4 Mean (SD) off and on medication scores for Activities of Daily Living (UPDRS part II) at baseline and at 12 months after surgery with stimulation on. ${ }^{* *} \mathrm{p}<0.001$, compared with the same condition before surgery.

Mobility, Emotional Well-being, Communication and Bodily Discomfort dimensions. Globally, bilateral STN DBS significantly improved quality of life by $14 \%$ as measured by the PDQ-39SI.

\section{Neuropsychometry}

Cognitive testing post operatively on 15 patients revealed few clinically relevant changes and did not suggest that the

Table 2 Timed motor tests in patients with bilateral deep brain stimulation of the subthalamic nucleus

\begin{tabular}{|c|c|c|c|c|}
\hline & \multicolumn{2}{|c|}{ Pre-operation } & \multicolumn{2}{|c|}{12 months post-operation } \\
\hline & Med off & Med on & Off & On \\
\hline Pronation-supination & $44.9(8.4)$ & $16.4(1.2)$ & $21.2(2.9)^{*} v$ & $17.3(2.0)$ \\
\hline Hand/arm movements & $20.12 .6)$ & $8.5(0.7)$ & $10.3(0.8)^{\star *}$ & $7.6(0.5)$ \\
\hline Finger dexterity & $55.1(6.0)$ & $32.8(4.1)$ & $41.8(5.7)^{\star *}$ & $32.6(3.2)$ \\
\hline Leg movements & $21.6(3.0)$ & $9.6(1.2)$ & $10.8(1.2)^{* *}$ & $8.3(0.8)$ \\
\hline Stand/walk & $34.2(8.2)$ & $16.2(7.6)$ & $13.3(2.3)^{*}$ & $13.0(4.0)$ \\
\hline
\end{tabular}

Values are expressed as means (SEM). The post-operative times shown above were with stimulation switched on ${ }^{*} p<0.05$; ${ }^{* *} p<0.01$ compared with the condition before surgery. 


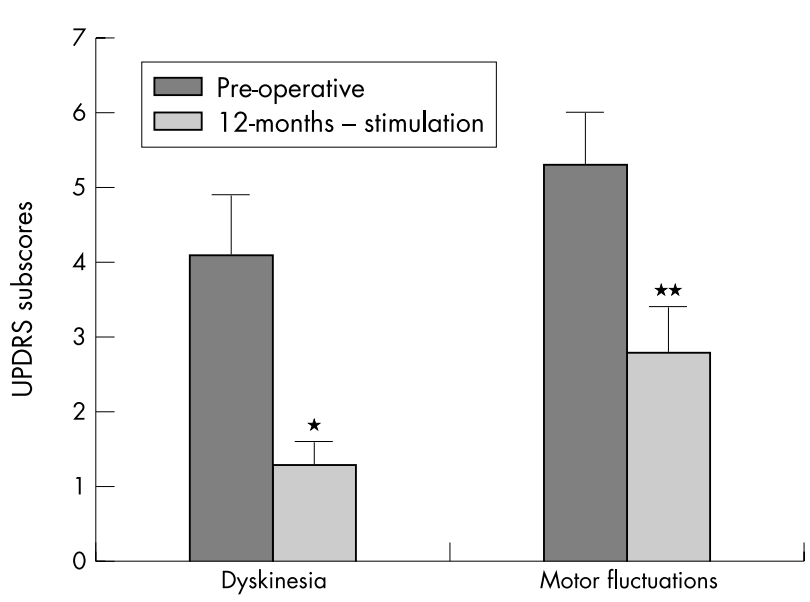

Figure 5 Mean (SD) scores for drug-induced dyskinesias and motor fluctuations before and 12 months after surgery with stimulation on. The subscores are for parts IVa and IVb of the Unified Parkinson's Disease Rating Scale respectively. ${ }^{*} p=0.006$; ${ }^{* *} p=0.003$, compared with the same condition before surgery.

procedure has significant adverse effects on cognition. A total of 204 individual test scores were available for analysis. According to the Reliable Change Index, $86 \%$ of the scores were either unchanged or improved at the 12 month postoperative follow up. Of the small number of scores that were "deteriorated", there was no systematic pattern of deterioration. There were relatively high rates of deteriorated scores on measures of verbal IQ, verbal memory retrieval, verbal and focused attention, and perseverance on a design fluency task. Between $20 \%$ and $29 \%$ of the scores on tests related to these cognitive functions were lower at the follow up assessment. However, these lowered scores were matched by an equivalent number of improved scores on the same measures for the remaining patients, suggesting no systematic effect of DBS on these neuropsychological domains. Certainly some of the changed scores will have reflected a chance occurrence. There remains the possibility that an idiosyncratic or iatrogenic negative effect of DBS may have occurred for individual

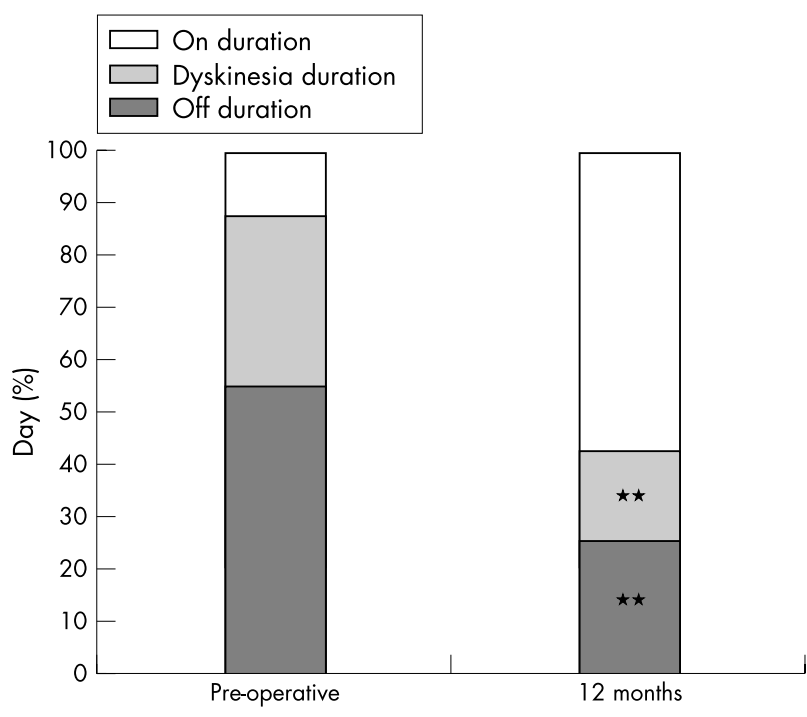

Figure 6 Mean on and off medication motor fluctuation durations before and 12 months after surgery. The dyskinesia and off period durations correspond to items 32 and 39 of part IV of the Unified Parkinson's Disease Rating Scale respectively. ${ }^{* *} p=0.01$, compared with the same condition before surgery.
Table 3 Effects of the bilateral STN DBS on QOL measured with PDQ-39 $(n=14)$

\begin{tabular}{|c|c|c|}
\hline & Baseline & 12 months \\
\hline Mobility & 78.8 & $51.3(-19 \%)$ \\
\hline Activities of daily living & 71.4 & $42.0(-30 \%)^{*}$ \\
\hline Emotional wellbeing & 40.8 & $25.6(-15 \%)$ \\
\hline Stigma & 47.3 & $25.9(-21 \%)^{*}$ \\
\hline Social support & 21.4 & $22.0(+1 \%)$ \\
\hline Cognitive impairment & 30.4 & $28.1(-2 \%)$ \\
\hline Communication & 45.8 & $33.3(-13 \%)$ \\
\hline Bodily discomfort & 57.2 & $42.3(-15 \%)$ \\
\hline PDQ-39 Summary Index & 48.1 & $34.5(-14 \%)^{\star *}$ \\
\hline \multicolumn{3}{|c|}{$\begin{array}{l}\text { Mean scores at baseline and at } 12 \text { months, with percentage change in } \\
\text { brackets at } 12 \text { months. } \\
\text { " } p<0.05 \text { compared with the condition before surgery (paired Student's } \\
t \text { test, after Bonferroni correction); }{ }^{* *} p<0.01 \text { compared with the condition } \\
\text { before surgery (paired Student's } t \text { test). }\end{array}$} \\
\hline
\end{tabular}

patients. Any such effect could only have been small as only two individuals showed deterioration across more than two tests. These individuals did not represent those with greater levels of impairment or cognitive vulnerability pre-operatively.

\section{Surgical procedure}

At surgery, perioperative macrostimulation was used for target confirmation and to optimise stimulator placement. For all cases, a single pass of the macrostimulating electrode was required for each side.

\section{Adverse effects}

In the 16 patients, there were no procedure or device related complications. Stimulation related complications included hypophonia in three patients and eyelid apraxia in two patients.

\section{DISCUSSION}

Our results are in accordance with other studies that have demonstrated that DBS of the STN is an effective treatment for patients suffering from advanced PD. Bilateral stimulation of STN greatly improved off period symptoms in these severely disabled patients. Adverse experiences were relatively few in our study cohort, and were all related to stimulation, including hypophonia in three cases and eyelid apraxia in two cases. Cognitive deterioration was detected in two of the patients, but as cognition in the remaining patients was mostly unchanged or improved, the decline in these two patients was solely progressive and presumably related to disease progression. Our results show that a predominantly anatomical MRI directed method without microelectrode recording can be used safely and effectively to deliver this treatment.

Deep brain stimulation of the STN resulted in a $61 \%$ reduction in UPDRS motor scores in the off medication state at 12 months. The cardinal parkinsonian symptoms (bradykinesia, rigidity, tremor, gait disturbances) were all significantly reduced by stimulation in the off medication state. On medication, motor scores were also significantly reduced by $40 \%$ with stimulation. The patients' functional performance was improved with stimulation, as demonstrated by significant improvements in the Activities of Daily Living scores (UPDRS part II and PDQ-39).

The use of high resolution T2 MR images in both the axial and coronal planes greatly facilitated the targeting of the dorsolateral STN. Within the STN, the dorsolateral portion of the nucleus is functionally implicated in the sensorimotor circuits, whereas the ventral portion is connected with associative areas, and the medial tip has connections with the limbic system. ${ }^{12}$ At surgery, perioperative macrostimulation 
was used for target confirmation and to optimise stimulator placement.

There have been several reports showing that bilateral STN stimulation is a very effective treatment for advanced PD. ${ }^{8}{ }^{19-23}$ The beneficial effects of STN stimulation are significant providing that the electrodes are placed appropriately. Most studies advocate the use of intraoperative microelectrode recording for verification of target localisation $^{24}$ and report an improvement in UPDRS motor scores of $58-68 \% .{ }^{6} 19212225$ In comparison, studies relying on intraoperative macrostimulation for target verification have reported a UPDRS motor score reduction of 36-68\%. ${ }^{23} 26-28$ Our finding of a $62 \%$ improvement is well above the median of the other studies using macrostimulation and within the range reported by studies using microelectrode recording. The mean baseline UPDRS motor score in our cohort (48) was slightly lower than that reported in the studies that used microelectrode recording (49.6-67.6). Compared with the other studies, we found a greater improvement in on period motor function; our on state improvement may in fact be an underestimate, as patients post-operatively had their on state UPDRS assessment after being given $200 \mathrm{mg}$ L-dopa, and in a number of cases this made patients dyskinetic, reducing their scores.

Most investigators experienced in performing STN surgery use microelectrode recording to delineate the boundaries of the target peroperatively to ensure optimal placement of the lesion or DBS lead. Microelectrode recording usually involves 3-6 recording tracts to be made before the lesioning or DBS lead is inserted. Nevertheless, the accuracy with which the target can be defined in the coronal plane depends upon the distance between the recording trajectories, which is typically $2 \mathrm{~mm}$. The maximal spatial resolution of the technique is therefore $2 \mathrm{~mm}$ and this may not be sufficiently precise to ensure optimal placement of a DBS lead (typically $1.3 \mathrm{~mm}$ in diameter) centrally within the STN (typically $3 \mathrm{~mm}$ in diameter). To compensate for the individual STN variations (as seen in figs 1 and 2) when using the indirect method with atlas-based coordinates, multiple microelectrode recording tracts would probably be required and the chance of accurately positioning the lead in the centre of the dorsolateral STN would be remote.

While side effects are not uncommon for both microelectrode recording and procedures guided without microelectrode recording, the rate of severe complications, such as intracerebral haematoma, appears to be higher when microelectrodes are used..$^{29} \mathrm{~A}$ review of the literature for procedures guided with and without miroelectrode recording found reported side effects to be greater among groups using microelectrode recording techniques. The available literature suggests that microelectrode recording techniques neither decrease risks nor increase targeting accuracy of ablative surgery or DBS procedures, compared with macrostimulation techniques. Unfortunately, to our knowledge, no formal trial comparing outcomes from a surgical team skilled in microelectrode targeting techniques with those from a team skilled in MRI targeting has been conducted. Furthermore, microelectrode recording techniques do not result in smaller lesions or in lower electrical parameters of DBS. An additional risk of using microelectrodes is that this technique undoubtedly prolongs the operating time compared with that of macroelectrodes, and may last up to $12 \mathrm{~h}$ for bilateral procedures, which may increase the risk of infection. The risk of brain shift, if no special measures are taken to avoid cerebrospinal fluid leak during surgery, will also increase, making target localisation more difficult. More importantly, this technique exposes patients to many hours of surgery awake, which for parkinsonian patients in an off state can be very distressing.
We acknowledge that the data presented here will not have ended the debate between proponents and opponents of microelectrode recording, and in an ideal world a randomised study comparing clinical outcomes from surgical interventions between teams expert in microelectrode recording techniques and teams expert in MRI based techniques would perhaps provide the definitive answer; however, as at present the results of such a study are not available, we hope that our data have added to the ongoing debate.

\section{CONCLUSIONS}

DBS of the STN is an effective treatment for patients with advance PD refractory to medical therapy, and results in significant reduction in patients' UPDRS motor scores in both off and on medication states. This treatment significantly reduces dyskinesias, motor fluctuations, and the requirement for L-dopa therapy, and significantly improves their functional performance (Activities of Daily Living). A predominantly anatomical MRI directed technique with macrostimulation can be used safely and effectively without the additional need for intraoperative microelectrode recording. In addition, our method was associated with an efficient use of operating time, and obviates the necessary costs of microelectrode recording.

\section{Authors' affiliations}

N K Patel, P Plaha, K O'Sullivan, R McCarter, P Heywood, S S Gill, Institute of Neurosciences, Frenchay Hospital, Bristol, UK

Competing interest: None declared

\section{REFERENCES}

1 Benabid AL, Benazzouz A, Hoffmann D, et al. Long-term electrical inhibition of deep brain targets in movement disorders. Movt Disord 1998; 13(Suppl 3): 119-25.

2 Krack P, Hamel W, Mehdorn HM, et al. Surgical treatment of Parkinson's disease. Curr Opin Neurol 1999;12:417-25.

3 Starr PA, Vitek JL, Bakay RA. Ablative surgery and deep brain stimulation for Parkinson's disease. Neurosurgery 1998; 43:989-1013; discussion 1013-15.

4 Benabid AL, Pollak P, Gervason C, et al. Long-term suppression of tremor by chronic stimulation of the ventral intermediate thalamic nucleus. Lancet 1991;337:403-6.

5 Benabid AL, Pollak P, Gao D, et al. Chronic electrical stimulation of the ventralis intermedius nucleus of the thalamus as a treatment of movement disorders. J Neurosurg 1996;84:203-14.

6 Limousin P, Krack P, Pollak P, et al. Electrical stimulation of the subthalamic nucleus in advanced Parkinson's disease. N Engl J Med 1998;339:1 105-11.

7 Siegfried J, Lippitz B. Bilateral chronic electrostimulation of ventroposterolateral pallidum: a new therapeutic approach for alleviating all parkinsonian symptoms. Neurosurgery 1994;35:1126-9; discussion 1129-30.

8 Deep-Brain Stimulation for Parkinson's Disease Study Group. Deep-brain stimulation of the subthalamic nucleus or the pars interna of the globus pallidus in Parkinson's disease. N Engl J Med 2001;345:956-63.

9 Starr PA, Vitek JL, DeLong M, et al. Magnetic resonance imaging-based stereotactic localization of the globus pallidus and subthalamic nucleus. Neurosurgery 1999;44:303-13, discussion 313-14.

10 Hutchison WD, Allan RJ, Opitz $\mathrm{H}$, et al. Neurophysiological identification of the subthalamic nucleus in surgery for Parkinson's disease. Ann Neurol 1998:44:622-8.

11 Schaltenbrand G, Bailey P. Einfuhrung in die stereotaktischen operationen mit einem atlas des menschlichen gehirn. Stuttgart: Thieme, 1959.

12 Parent A. Basal ganglia. In: Parent A, ed. Carpenter's human neuroanatomy. Baltimore: Williams and Wilkins, 1996:838-43.

13 Langston JW, Widner H, Goetz CG, et al. Core assessment program for intracerebral transplantations (CAPIT). Mov Disord 1992;7:2-13.

14 McCarter RJ, Walton NH, Rowan AF, et al. Cognitive functioning after subthalamic nucleotomy for refractory Parkinson's disease. J Neurol Neurosurg Psychiatry 2000;69:60-6.

15 Jacobson NSTTP. Clinical significance: a statistical approach to defining meaningful change in psychotherapy research. J Consul Clin Psychol $1991 ; 19: 889-896$.

16 Trepanier LLS-CJ, Lozano AM, Lang AE. Neuropsychological consequences of posteroventral pallidotomy for the treatment of Parkinson's disease. Neurology 1998;51:207-15.

17 Troster Al FJ, Wilkinson SB, Pahwa R, et al. Unilateral pallidal stimulation for Parkinson's disease: neurobehavioural functioning before and three months after electrode implantation. Neurology 1997;49:1078-83. 
18 Pahwa R, Wilkinson S, Smith D etal. High-frequency stimulation of the globus pallidus for the treatment of Parkinson's disease. Neurology 1997;49:249-53.

19 Fraix V, Pollak P, Van Blercom N, et al. Effect of subthalamic nucleus stimulation on levodopa-induced dyskinesia in Parkinson's disease. Neurology 2000:55:1921-3.

20 Limousin P, Pollak P, Benazzouz A, et al. Bilateral subthalamic nucleus stimulation for severe Parkinson's disease. Mov Disord 1995;10:672-4.

21 Molinuevo JL, Valldeoriola F, Tolosa E, et al. Levodopa withdrawal after bilateral subthalamic nucleus stimulation in advanced Parkinson disease. Arch Neurol 2000:57:983-8.

22 Houeto JL, Damier P, Beijani PB, et al. Subthalamic stimulation in Parkinson disease: a multidisciplinary approach. Arch Neurol 2000;57:461-5.

23 Pinter MM, Alesch F, Murg M, et al. Deep brain stimulation of the subthalamic nucleus for control of extrapyramidal features in advanced idiopathic parkinson's disease: one year follow-up. J Neural Transm 1999; 106:693-709.

24 Guridi J, Rodriguez-Oroz MC, Lozano AM, et al. Targeting the basal ganglia for deep brain stimulation in Parkinson's disease. Neurology 2000;55(Suppl 6): $521-8$
25 Kumar R Lozano AM, Kim YJ, et al. Double-blind evaluation of subthalamic nucleus deep brain stimulation in advanced Parkinson's disease. Neurology 1998;51:850-5.

26 Moro E, Scerrati M, Romito LM, et al. Chronic subthalamic nucleus stimulation reduces medication requirements in Parkinson's disease. Neurology 1999:53:85-90.

27 Burchiel KJ, Anderson VC, Favre J, et al. Comparison of pallidal and subthalamic nucleus deep brain stimulation for advanced Parkinson's disease: results of a randomized, blinded pilot study. Neurosurgery 1999:45:1375-82; discussion 1382-4.

28 Volkmann J, Allert N, Voges J, et al. Safety and efficacy of pallidal or subthalamic nucleus stimulation in advanced PD. Neurology 2001;56:548-51.

29 Carroll CB, Scott R, Davies LE, et al. The pallidotomy debate. Br J Neurosurg 1998; 12:146-50.

30 Hariz MI, Fodstad H. Do microelectrode techniques increase accuracy or decrease risks in pallidotomy and deep brain stimulation? A critical review of the literature. Stereotact Funct Neurosurg 1999:72:157-69.

\section{Clinical Evidence - Call for contributors}

Clinical Evidence is a regularly updated evidence based journal available worldwide both as a paper version and on the internet. Clinical Evidence needs to recruit a number of new contributors. Contributors are health care professionals or epidemiologists with experience in evidence based medicine and the ability to write in a concise and structured way.

\section{Currently, we are interested in finding contributors with an interest in the following clinical areas:}

Altitude sickness; Autism; Basal cell carcinoma; Breast feeding; Carbon monoxide poisoning; Cervical cancer; Cystic fibrosis; Ectopic pregnancy; Grief/bereavement; Halitosis; Hodgkins disease; Infectious mononucleosis (glandular fever); Kidney stones; Malignant melanoma (metastatic); Mesothelioma; Myeloma; Ovarian cyst; Pancreatitis (acute); Pancreatitis (chronic); Polymyalgia rheumatica; Post-partum haemorrhage; Pulmonary embolism; Recurrent miscarriage; Repetitive strain injury; Scoliosis; Seasonal affective disorder; Squint; Systemic lupus erythematosus; Testicular cancer; Varicocele; Viral meningitis; Vitiligo However, we are always looking for others, so do not let this list discourage you.

Being a contributor involves:

- Appraising the results of literature searches (performed by our Information Specialists) to identify high quality evidence for inclusion in the journal.

- Writing to a highly structured template (about 2000-3000 words), using evidence from selected studies, within 6-8 weeks of receiving the literature search results.

- Working with Clinical Evidence Editors to ensure that the text meets rigorous epidemiological and style standards.

- Updating the text every eight months to incorporate new evidence.

- Expanding the topic to include new questions once every 12-18 months.

If you would like to become a contributor for Clinical Evidence or require more information about what this involves please send your contact details and a copy of your CV, clearly stating the clinical area you are interested in, to Claire Folkes (cfolkes@bmigroup.com).

\section{Call for peer reviewers}

Clinical Evidence also needs to recruit a number of new peer reviewers specifically with an interest in the clinical areas stated above, and also others related to general practice. Peer reviewers are health care professionals or epidemiologists with experience in evidence based medicine. As a peer reviewer you would be asked for your views on the clinical relevance, validity, and accessibility of specific topics within the journal, and their usefulness to the intended audience (international generalists and health care professionals, possibly with limited statistical knowledge). Topics are usually 2000-3000 words in length and we would ask you to review between 2-5 topics per year. The peer review process takes place throughout the year, and our turnaround time for each review is ideally 10-14 days.

If you are interested in becoming a peer reviewer for Clinical Evidence, please complete the peer review questionnaire at www.clinicalevidence.com or contact Claire Folkes(cfolkes@bmigroup.com). 